\title{
A Low-power Digital Filter IC via Soft DSP $\dagger$
}

\author{
Rajamohana Hegde and Naresh R. Shanbhag \\ ECE Department/ Coordinated Science Laboratory \\ University of Illinois at Urbana-Champaign, Urbana, IL 61801. \\ email:[rhegde, shanbhag]@mail.icims.csl.uiuc.edu
}

\begin{abstract}
In this paper we present an integrated circuit implementation of a soft DSP based low-power digital filter in $0.35 \mu \mathrm{m}, 3.3 \mathrm{~V}$ CMOS process. Soft DSP is a low-power technique that employs voltage overscaling (VOS) and algorithmic noise-tolerance (ANT) to push the limits of energy-efficiency beyond that achievable by voltage scaling alone. VOS refers. to scaling the supply voltage beyond the limit imposed by the throughput constraints. ANT is an algorithmic level error-control technique that is employed to restore the algorithmic performance degradation in terms of output signal-to-noise ratio $(S N R)$ caused by VOS. Measured results indicate $40 \%-67 \%$ reduction in energy dissipation over optimally voltagescaled systems with less than $1 d b$ loss in $S N R$ for a wide range of filter bandwidths $\left(0.05 f_{s}-0.25 f_{s}\right.$, where $f_{s}$ is the sampling frequency).
\end{abstract}

\section{Introduction}

Supply voltage scaling has proved to be an effective technique [1] for designing low-power systems, in general, and digital signal processing (DSP) and communications systems, in particular. However, a reduction in supply voltage results in an increase in the circuit delay given by

$$
\tau_{d}=\frac{C_{L} V_{d d}}{\beta\left(V_{d d}-V_{t}\right)^{\alpha}}
$$

where $\alpha$ is the velocity saturation index, $\beta$ is the device transconductance, and $V_{t}$ is the device threshold voltage. Low-power design techniques where throughput is traded off for energy-efficiency via voltage scaling in either static [2] or dynamic fashion [3] have been proposed. Thus, for all known techniques, the extent of voltage scaling (and hence power savings) is limited by the throughput requirements of the application.

This paper proposes soft DSP as a technique for overcoming this limit and thereby achieving even greater power savings (up to $70 \%$ more than the best achievable today without soft DSP). Soft DSP employs voltage overscaling (VOS) and algorithmic noise-tolerance

${ }^{\dagger}$ This work was supported by NSF grant CCR 00-00987.
(ANT) and is particularly suitable for the design of lowpower DSP and communications systems as described next.

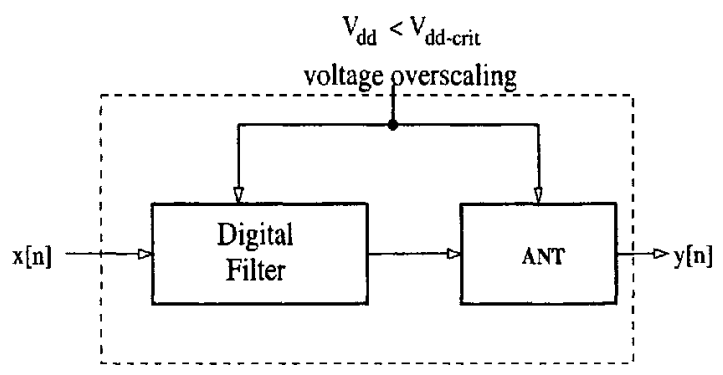

Figure 1: Soft DSP

Figure 1 shows the key idea behind soft DSP. The input $x[n]$ arrives at a fixed sample rate $f_{s}$. This puts an upper bound equal to the sample period $T_{s}=1 / f_{s}$ on the critical path delay $T_{c p}$ of the digital filter. The supply voltage at which $T_{c p}=T_{s}$ is denoted by $V_{d d, c r i t}$, which is also the lower bound on the supply voltage $V_{d d}$. Present day voltage scaling operates in the range $V_{d d} \geq V_{d d \text {,crit }}$. VOS, on the other hand, implies operation in the range $V_{d d}<V_{d d, c r i t}$ resulting in greater energy savings but also leading to intermittent errors at the output whenever the critical paths are excited. In the context of DSP and communication systems, these errors lead to a degradation in the signal-to-noise ratio $(S N R)$. Thus, voltage overscaled systems need to include some form of error-control to restore the $S N R$ degradation. The error-control block should also have low complexity as compared to the digital filter in order for appreciable energy savings to occur. It turns out that one can employ ANT [4] techniques to design low-complexity error-control blocks for DSP and communication systems. This paper demonstrates the energy savings via soft DSP (see Figure 1).

In this paper, we describe measured results quantifying the energy savings of a prototype soft digital filter IC designed and fully tested in a $0.35 \mu \mathrm{m}, 3.3 \mathrm{~V}$ CMOS process. Measured results indicate $40 \%-67 \%$ reduction in energy dissipation over optimally voltage-scaled present day systems with less than $1 d b$ loss in $S N R$ for a wide 
range of filter bandwidths $\left(0.05 f_{s}-0.25 f_{s}\right.$, where $f_{s}$ is the sampling frequency).

The rest of this paper is organized as follows. In the next section, we present the ANT scheme for digital filtering. Then the architecture of the soft filter implementation is presented followed by the implementation details and measured results.

\section{Algorithmic Noise-Tolerance (ANT)}

The ANT technique for filtering proposed in [4] is shown in Figure 2. This algorithm exploits the correlation in the filter output to perform error-control. A linear predictor is employed at the filter output to predict the current filter output based on the past outputs. The current filter output is then compared to the predicted value. If the difference between the predicted and the actual sample is small then no error is said to have occurred. If the difference is larger than a set threshold, an error is declared and the predicted sample is used as the actual filter output.

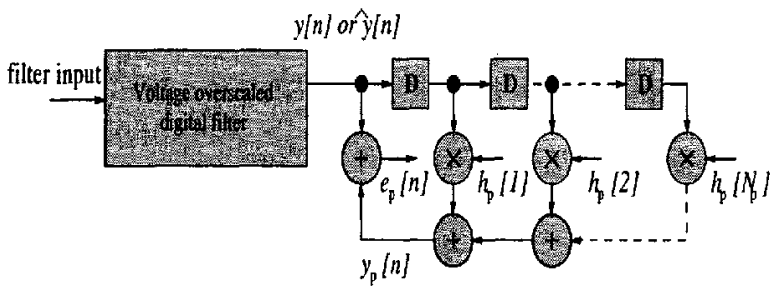

Figure 2: Prediction-based ANT scheme for filtering.

The output of a digital FIR filter shown in Fig. 2, when the filter is error-free, is denoted by $y[n]$ and is given by,

$$
y[n]=\sum_{k=0}^{N-1} h[k] x[n-k],
$$

where $h[k]$ denotes the filter impulse response, $x[n]$ is the filter input and $N$ is the number of taps in the filter. The filter output $\hat{y}[n]$ when the filter is operating under VOS is given by

$$
\hat{y}[n]=y[n]+y_{\text {err }}[n],
$$

where $y_{e r r}[n]$ denotes the error in the filter output due to errors. Note that $y_{\text {err }}[n]$ is non-zero only when the input pattern is such that either the critical path or other longer paths in the filter implementation are excited.

If $y_{p}[n]$ is the output of the predictor, then the prediction error $e_{p}[n]$, when the filter is noiseless, is given by

$$
e_{p}[n]=y[n]-y_{p}[n] .
$$

The minimum mean square error (MMSE) depends on the autocorrelation function of $y[n]$ and the order of the predictor. Let $\hat{y}[n], \hat{y_{p}}[n]$, and $\hat{e_{p}}[n]$ denote the filter output, the predictor output, and the prediction error, respectively, in presence of soft errors. It can be shown that [4]

$$
\hat{e_{p}}[n]=y_{e r r}[n]+e_{p}[n] .
$$

Assuming that no more errors occur in the next $N_{p}$ output samples, we have show that [4],

$$
\hat{e_{p}}[n+m]=-h_{p}[m] y_{e r r}[n]+e_{p}[n+m]
$$

for $m=1, \cdots, N_{p}$.

The ANT schemes presented in [4] are based on the fact that, in absence of errors, the magnitude of the prediction error is small. From (5) and (6), it can be seen that a large $y_{\text {err }}(n)$ (when it is non-zero), leads to a large difference between $e_{p}[n]$ and $\hat{e_{p}}[n]$. This leads to efficient error detection. In presence of an error due to overscaling, the magnitude of the prediction error increases substantially as the errors occur in the most significant bits (MSBs). In case an error is detected, the predictor output corresponding to the erroneous sample is resolved to be the actual filter output.

The prediction-based ANT algorithm can provide effective error control if the following three conditions are satisfied: 1.) errors in $y[n]$ are spaced apart by at least $2 N_{p}$ samples, 2.) the error magnitudes are large, and 3.) the error-control block is error free. These three conditions can be easily met in practice by employing an architecture which has the following properties: 1.) it is delay-imbalanced, 2.) employs arithmetic units that compute in an LSB-first manner, and 3.) the errorcontrol block has a shorter critical path than that of the filter. All three properties are present in an architecture that employs ripple-carry adders, array multipliers and the prediction-based ANT technique.

\section{Chip Architecture}

The architecture of the prototype VOS digital filter IC is shown in Figure 3. The IC has two multiply-accumulate (MAC) circuits, one for the digital FIR filter (FMAC) and the other for the predictor in the error control block (ECMAC) as shown in Figure 3. The FMAC consists of an $10 b \times 8 b$ unsigned array multiplier followed by a modified 2's complement $22 b$ accumulator. The ECMAC is architecturally similar to the FMAC but has a $5 b \times$ $8 b$ array multiplier followed by a $16 b$ accumulator. The smaller precisions of the ECMAC translate to a lesser area and power overhead and also a shorter critical path delay.

The coefficient as well as data inputs to the FMAC and the coefficient inputs to the ECMAC are fed externally and are latched. Note that the clock signal that drives the ECMAC (fast clock) latches is slower than that for the 


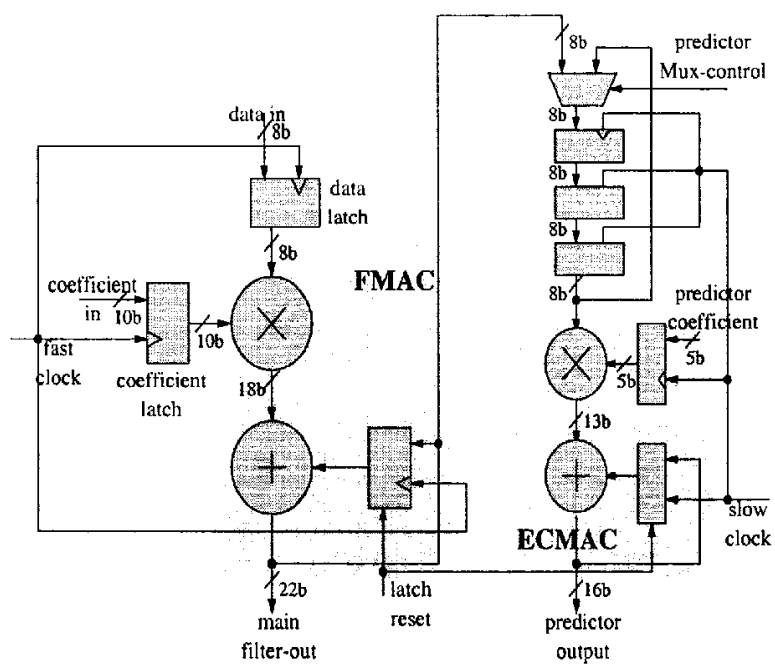

Figure 3: The chip architecture.

FMAC (slow clock). This is because the length of the predictor is substantially shorter than that of the FIR filter. For a filter length of $N=32$ taps, the maximum predictor length is $N_{p}=4$ taps. For the experiments reported in this paper, the fast clock was 8 times faster than the slow clock. This ensures correct operation of the ECMAC at reduced supply voltages due to the slower clock and hence enables VOS for both FMAC as well as ECMAC.

The FMAC output feeds into the ECMAC which employs the past FMAC output samples to statistically predict the present FMAC output. All circuit structures are implemented for most part in static CMOS except for the latches which employ the $C^{2} M O S$ style. The fulladders in both the FMAC as well as the ECMAC are implemented in the classic 24-transistor symmetric configuration [6]. The latches are powered with a separate supply that is held constant.

\section{Experimental results}

A prototype VOS digital filter IC (Figure 4) has been designed and fully tested in a $0.35 \mu \mathrm{m}, 3.3 \mathrm{~V}$ CMOS process. The transistor complexity of the FMAC is 3872 while that of the ECMAC is 2423. Measurements on the test bench have shown that the critical supply voltage for FMAC at a clock frequency of $88 \mathrm{MHz}$ is $V_{d d, c r i t, F M A C}=3.55 \mathrm{~V}$. The worst-case power consumed at this frequency and voltage is $105.47 \mathrm{~mW}$. Due to the lower precision and fewer taps in the predictor in Figure. 3 , the ECMAC is clocked at a frequency of $11 \mathrm{MHz}$ which is $8 X$ slower than the main MAC. Thus, the ECMAC has $V_{d d, \text { crit }, E C M A C}=2.25 \mathrm{~V}$.

In the VOS mode, the FMAC and ECMAC are oper-

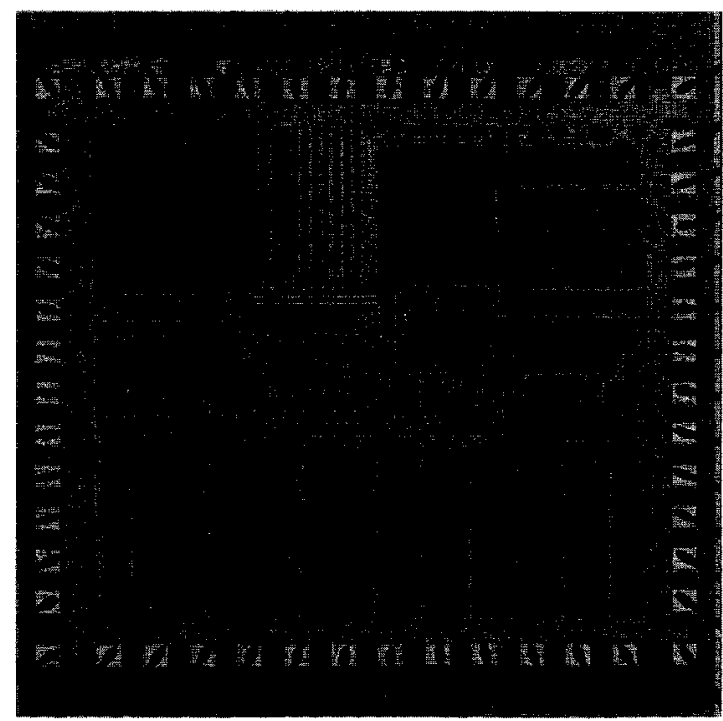

Figure 4: Chip micrograph.

ated at the same voltage which lies in the sub-critical range $2.32 \mathrm{~V}<V_{d d, F M A C}<V_{d d, c r i t, F M A C}$ at a fixed clock frequency of $88 \mathrm{MHz}$. Thus, the FMAC has intermittent errors at the output while the EMAC is errorfree. The algorithmic performance in terms of $S N R$ is

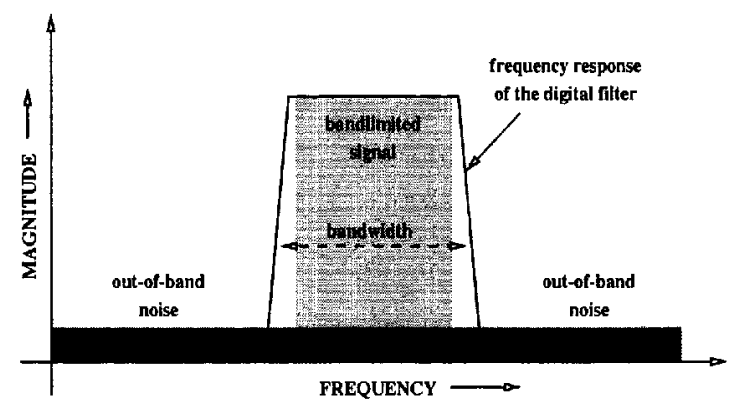

Figure 5: Frequency selective filtering.

measured in the context of a frequency selective filtering application as illustrated in Figure 5. Note that the signal is bandlimited and the noise is wideband. The digital filter is employed to suppress the out-of-band noise and improve the $S N R$. The filter bandwidth is set to be the same as the signal bandwidth. This ensures maximum out-of-band noise rejection. The coefficients of the filter determine its frequency response. For the implementation presented in this paper, the filter coefficients are fed externally. This enables measurements for any bandwidth.

The plot of $S N R$ vs. measured power savings for filter bandwidths ranging from $0.05 f_{s}-0.25 f_{s}$ is shown in 
Figure 6. The power savings is given by

$$
P S=\frac{P_{\text {ref }}-P_{v o s}}{P_{\text {ref }}},
$$

where $P_{\text {vos }}$ is the power at overscaled supply voltage. and $P_{\text {ref }}$ is the power measured respect to a reference filter operating at $V_{d d, c r i t, F M A C}=3.55 \mathrm{~V}$. The reference filter is referred to as an optimally-scaled present day system because this filter operates error-free and hence does not require ANT techniques. The power consumed by the ECMAC is included in power calculations for the VOS filter. Observe from Figure 6 that power savings in the range $40 \%-67 \%$ is achieved over a present day voltage scaling scenario with a degradation of less than $1 \mathrm{~dB}$ in output $S N R$ for a 32-tap FIR filter for bandwidths in the range $0.05 f_{s}-0.25 f_{s}$ at a clock speed of $88 \mathrm{MHz}$. As expected, an increase in bandwidth reduces power savings due to the reduction in output correlation.

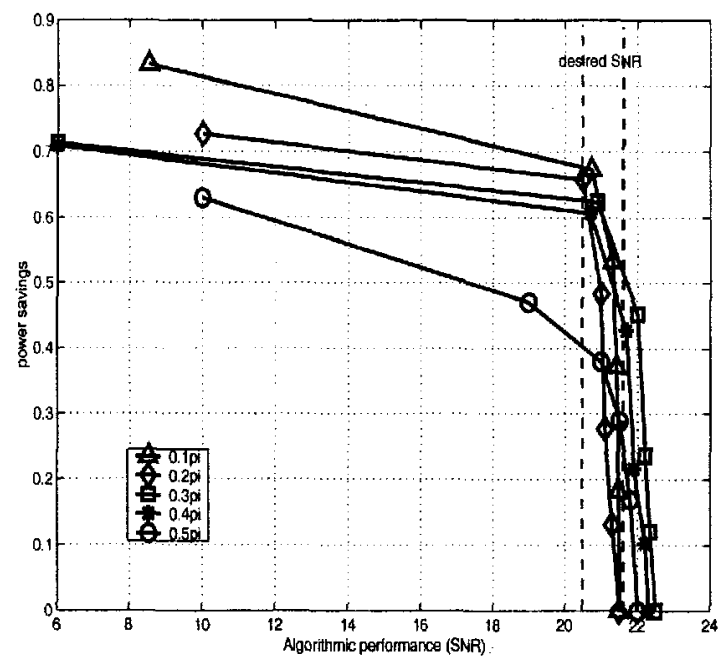

Figure 6: Measured results: Algorithmic performance vs. Power savings.

\section{Conclusions}

In this paper, we have presented an IC implementation of a low-power digital filter. Voltage overscaling (VOS) in combination with algorithmic noise-tolerance (ANT) [4] (referred to as soft DSP) is the low-power technique being employed. We have demonstrated experimentally that the proposed low-power technique provides up to $67 \%$ additional energy savings over an optimally voltagescaled present day system, i.e., a system that operates with a supply voltage equal to $V_{d d, \text { crit }}$.

The technique described in this paper opens up new areas of research within low-power design such as the investigation of 1.) arithmetic and filter architectures that favor soft DSP. and 2.) ANT techniques for other commonly employed DSP blocks such as FFTs, DCTs, IIR and adaptive filters. In 1.), the goal is to design architectures that have a graceful degradation property under VOS, while in 2.) the key is to develop effective techniques, i.e., techniques in which error-control complexity is small compared to that of the main block while providing maximum error correction. Due to the energy-delay relationship inherent in integrated circuits, one can employ ANT for improving the throughput as well. On a broader scale, apart from low-power design, ANT techniques have applications in improving the reliability of integrated microsystems in current and future technologies by being able to combat deep submicron noise and process non-idealities at the algorithmic level. More importantly, ANT techniques enable a seamless trade-off between energy-efficiency/throughput vs, reliability which is an important asset to have as feature-sizes scale into the sub-0.1 $\mu m$ range.

\section{References}

[1] A. Chandrakasan and R. W. Brodersen, "Minimizing power consumption in digital CMOS circuits," Proceedings of the IEEE, vol. 83, no. 4, pp. 498-523, April 1995.

[2] R. Gonzalez, et. al., "Supply and threshold voltage scaling for low power CMOS," IEEE Journal of Solid State Circuits, vol. 32, No. 8, Aug. 1997.

[3] A. Sinha and A. Chandrakasan, "Energy efficient filtering using adaptive precision and variable voltage," Proceedings of 1999 ASIC/SOC Conference, pp. 327331, 1999.

[4] R. Hegde and N. R. Shanbhag, "Energy efficient signal processing via algorithmic noisetolerance," Proceedings of 1999 International Symposium on Low Power Electronics and Design, pp. 3035, August, 1999.

[5] J. G. Proakis and D. G. Manolakis, Digital Signal Processing: Principles, Algorithms and Applications Prentice-Hall, New Jersey, 1996.

[6] J. M. Rabaey, Digital Integrated Circuits: A Design Perspective. Prentice-Hall, New Jersey, 1996. 